\title{
Conformational Flexibility and Crystallization: The Case of 4-hexyloxybenzoic Acid
}

Wei Du ${ }^{\dagger}$, Huifen Wang ${ }^{\dagger}$, Runpu Wang ${ }^{\dagger}$, Xuemin Wang ${ }^{\dagger}$, Penggao Cheng ${ }^{\dagger}$, Jianping

$$
\text { Zhang }^{\dagger}, \text { Na Tang }{ }^{\dagger, *}, \text { Liang } \mathrm{Zhu}^{\dagger, *}, \text { Penglei Cuit }
$$

${ }^{\dagger}$ College of Chemical Engineering and Materials Science, Tianjin Key Laboratory of Brine Chemical Engineering and Resource Eco-utilization, Tianjin University of Science \& Technology, Tianjin 300457, People's Republic of China

${ }^{\ddagger}$ State Key Laboratory of Multiphase Complex Systems, Institute of Process Engineering, Chinese Academy of Sciences, Beijing 100190, People's Republic of China

*Email: titangna@ tust.edu.cn; zhuliang@tust.edu.cn

\section{Supplementary Information}

The measured solubility of HOBA Form I in different solvents and the dissolving properties deduced from Van't Hoff expression are listed in Table S1, Table S2 and Table S3. The polymorph outcome and induction time of fast cooling crystallization, evaporative crystallization and anti-solvent crystallization are shown in Table S4, Table S5 and Table S6. The IR, Raman and Hot Stage identification of HOBA Form I and Form II are shown in Figure S1, Figure S2, Figure S3 and Figure S4. The fitted curves of the solubility via Van't Hoff expression is shown in Figure S5.

According to Van’t Hoff expression equation (S1), dissolution enthalpy $\left(\Delta_{d i s} H^{\circ}\right)$ and entropy $\left(\Delta_{d i s} S^{\circ}\right)$ of HOBA in binary mixed solvent can be calculated from the slope 
and intercept of linear $\ln x$ versus $1 / T$ plot.

$$
\ln x=-\frac{\Delta_{\mathrm{dis}} H^{\circ}}{\mathrm{R} T}+\frac{\Delta_{\mathrm{dis}} S^{\circ}}{\mathrm{R}}
$$

where $R=8.3145 \mathrm{~J} \cdot \mathrm{K}^{-1} \cdot \mathrm{mol}^{-1}$, represents the gas constant, and $T$ represents the experimental temperature.

The Gibbs-Helmholtz equation can be used to calculate dissolution Gibbs energy $\left(\Delta_{d i s} G^{\circ}\right)$ of FFA in the mixed solvent, as following,

$$
\Delta_{\text {dis }} G^{\circ}=\Delta_{\text {dis }} H^{\circ}-T \Delta_{\text {dis }} S^{\circ}
$$

To compare the relative contribution of enthalpy and entropy to the standard Gibbs energy during the dissolution process, $\% \xi H$ and $\% \xi S$ are defined as Eq. (S3) and Eq. (S4), as have been mentioned in references.

$$
\begin{gathered}
\% \xi H=\frac{\left|\Delta_{d i s} H^{\circ}\right|}{\left|\Delta_{d i s} H^{\circ}\right|+\left|T \Delta_{d i s} S^{\circ}\right|} \times 100 \\
\% \xi S=\frac{\left|T \Delta_{d i s} S^{\circ}\right|}{\left|\Delta_{d i s} H^{\circ}\right|+\left|T \Delta_{d i s} S^{\circ}\right|} \times 100
\end{gathered}
$$

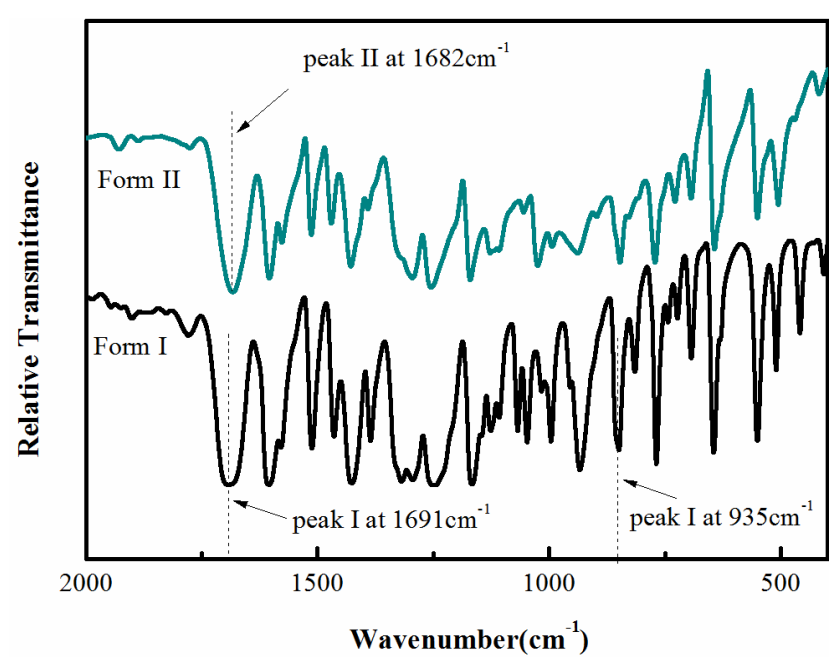

Figure S1. The FTIR spectra of HOBA polymorphs 


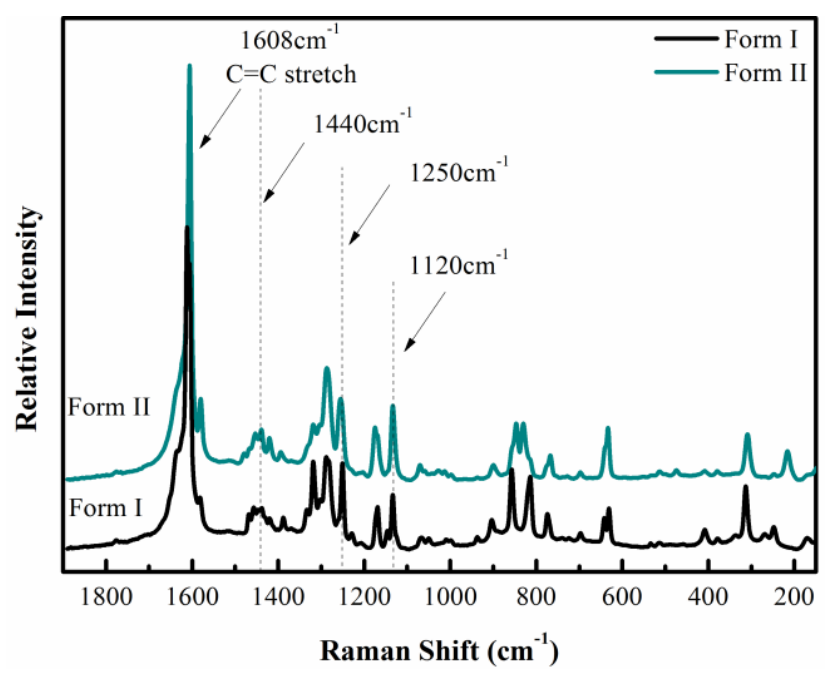

(a)

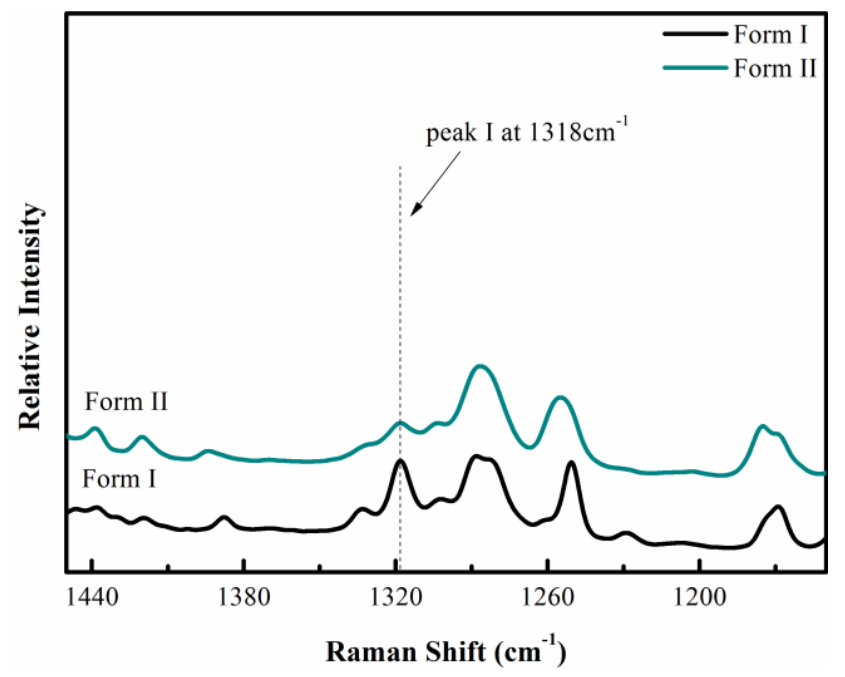

(b) 


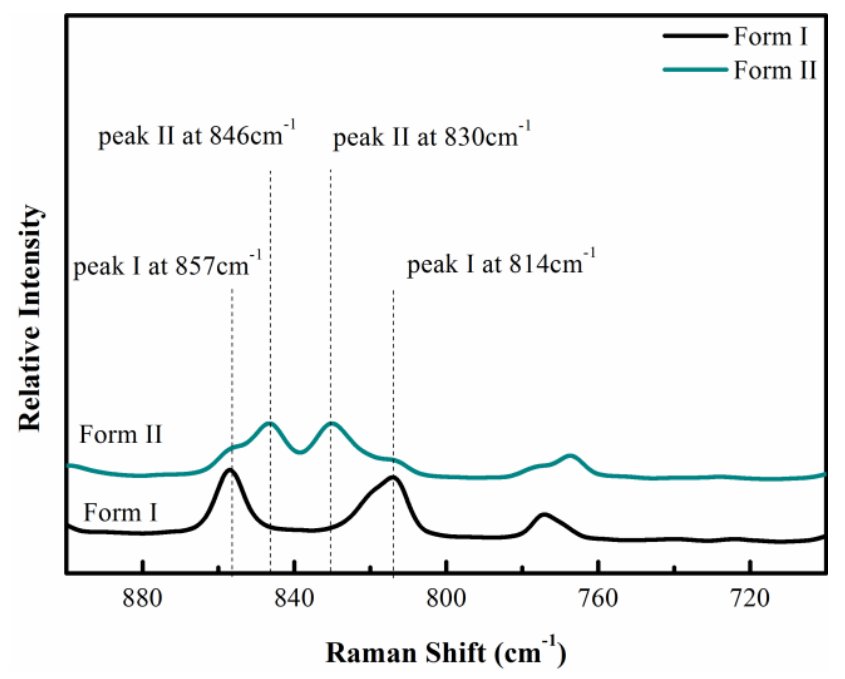

(c)

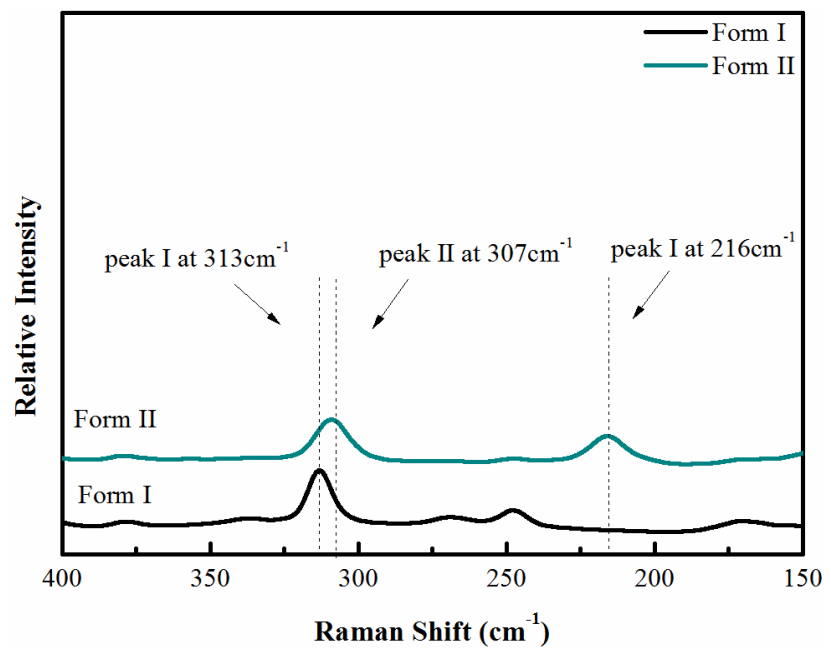

(d)

Figure S2. Raman spectra of Form I and Form II of HOBA: (a) 150-1950 $\mathrm{cm}^{-1}$

(b) $1150-1450 \mathrm{~cm}^{-1}$ (c) $700-900 \mathrm{~cm}^{-1}$ (d) $150-400 \mathrm{~cm}^{-1}$ 


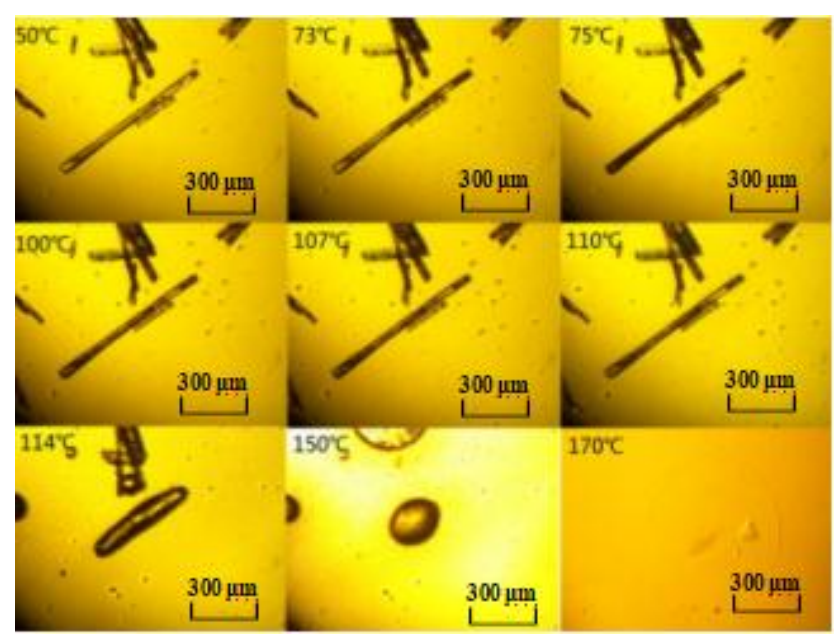

Figure S3. HSM snapshots of HOBA Form I

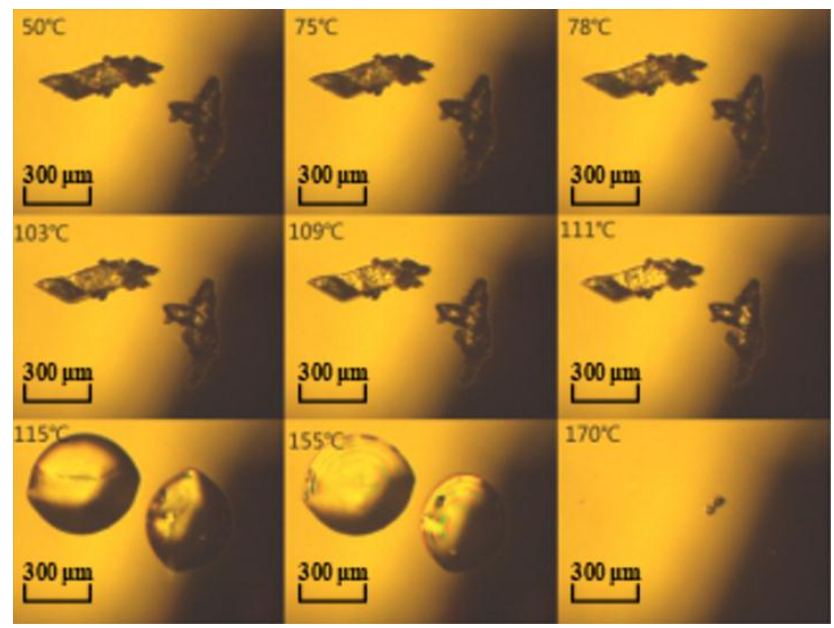

Figure S4. HSM snapshots of HOBA Form II 


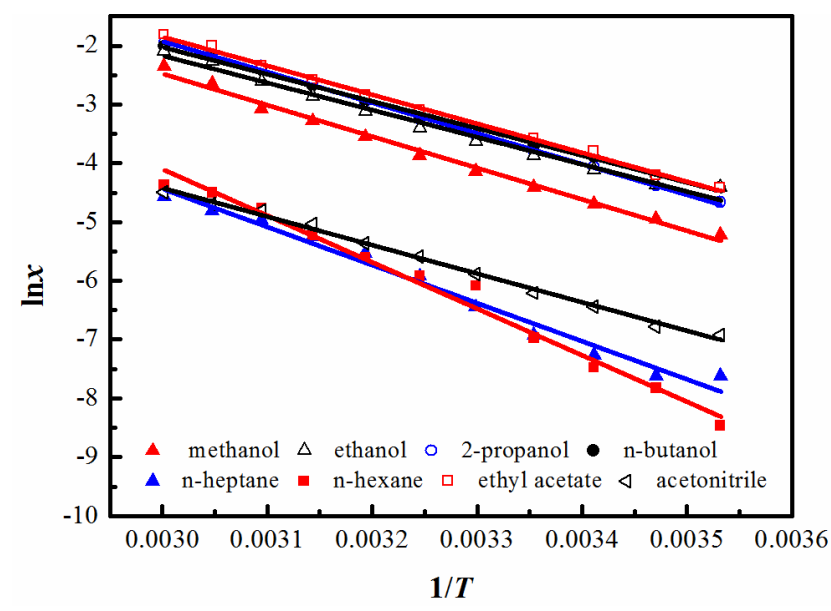

Figure S5. The vant Hoff plots of $\ln x$ versus $1 / T$ in different pure organic solvents.

Table S1. Experimental and calculated mole fraction solubility of 4-hexyloxybenzoic acid in different pure solvents from $283.15 \mathrm{~K}$ to $333.15 \mathrm{~K}$ at $0.10 \mathrm{MPa}$.

\begin{tabular}{cccccccc}
\hline$T / \mathrm{K}$ & $10^{2} x$ & SD & $10^{2} x^{\text {cal,Apel }}$ & $T / \mathrm{K}$ & $10^{2} x$ & SD & $10^{2} x^{\text {cal,Apel }}$ \\
\hline methanol & & & \multicolumn{5}{c}{ ethyl acetate } \\
283.15 & 0.543 & $1.037 \mathrm{E}-05$ & 0.599 & 283.15 & 1.198 & $3.307 \mathrm{E}-05$ & 1.202 \\
288.15 & 0.709 & $1.485 \mathrm{E}-05$ & 0.744 & 288.15 & 1.488 & $2.205 \mathrm{E}-05$ & 1.570 \\
293.15 & 0.920 & $8.576 \mathrm{E}-06$ & 0.939 & 293.15 & 2.251 & $3.225 \mathrm{E}-05$ & 2.048 \\
298.15 & 1.212 & $7.415 \mathrm{E}-05$ & 1.203 & 298.15 & 2.804 & $9.933 \mathrm{E}-06$ & 2.669 \\
303.15 & 1.604 & $4.110 \mathrm{E}-06$ & 1.563 & 303.15 & 3.374 & $6.944 \mathrm{E}-06$ & 3.476 \\
308.15 & 2.086 & $3.593 \mathrm{E}-05$ & 2.057 & 308.15 & 4.499 & $5.715 \mathrm{E}-06$ & 4.523 \\
313.15 & 2.886 & $8.576 \mathrm{E}-06$ & 2.739 & 313.15 & 5.789 & $2.449 \mathrm{E}-05$ & 5.878 \\
318.15 & 3.769 & $4.989 \mathrm{E}-06$ & 3.687 & 318.15 & 7.552 & $1.866 \mathrm{E}-04$ & 7.630 \\
323.15 & 4.566 & $7.225 \mathrm{E}-04$ & 5.016 & 323.15 & 9.574 & $4.205 \mathrm{E}-05$ & 9.893 \\
328.15 & 7.067 & $8.532 \mathrm{E}-05$ & 6.887 & 328.15 & 13.460 & $5.070 \mathrm{E}-05$ & 12.812 \\
333.15 & 9.531 & $1.453 \mathrm{E}-04$ & 9.538 & 333.15 & 16.321 & $2.274 \mathrm{E}-04$ & 16.570 \\
ethanol & & & & acetonitrile & & & \\
283.15 & 1.207 & $1.143 \mathrm{E}-05$ & 0.986 & 283.15 & 0.099 & $1.594 \mathrm{E}-05$ & 0.071 \\
288.15 & 1.273 & $1.452 \mathrm{E}-05$ & 1.264 & 288.15 & 0.114 & $1.306 \mathrm{E}-05$ & 0.106 \\
293.15 & 1.628 & $1.594 \mathrm{E}-05$ & 1.623 & 293.15 & 0.158 & $1.268 \mathrm{E}-05$ & 0.153 \\
298.15 & 2.079 & $7.483 \mathrm{E}-06$ & 2.088 & 298.15 & 0.201 & $1.228 \mathrm{E}-05$ & 0.214 \\
303.15 & 2.652 & $2.944 \mathrm{E}-06$ & 2.691 & 303.15 & 0.278 & $1.686 \mathrm{E}-05$ & 0.291 \\
308.15 & 3.331 & $1.812 \mathrm{E}-04$ & 3.472 & 308.15 & 0.372 & $2.155 \mathrm{E}-05$ & 0.386 \\
313.15 & 4.451 & $5.123 \mathrm{E}-04$ & 4.484 & 313.15 & 0.473 & $4.320 \mathrm{E}-06$ & 0.499 \\
318.15 & 5.715 & $7.838 \mathrm{E}-05$ & 5.797 & 318.15 & 0.664 & $1.164 \mathrm{E}-04$ & 0.630 \\
323.15 & 7.353 & $8.981 \mathrm{E}-05$ & 7.499 & 323.15 & 0.815 & $4.532 \mathrm{E}-05$ & 0.779 \\
328.15 & 10.296 & $5.144 \mathrm{E}-05$ & 9.706 & 328.15 & 0.917 & $1.025 \mathrm{E}-04$ & 0.942 \\
333.15 & 12.292 & $3.266 \mathrm{E}-05$ & 12.568 & 333.15 & 1.119 & $5.552 \mathrm{E}-05$ & 1.118 \\
$2-$-propanol & & & & $n-h e x a n e$ & & & \\
283.15 & 0.943 & $2.055 \mathrm{E}-06$ & 0.785 & 283.15 & 0.023 & $2.859 \mathrm{E}-05$ & 0.052
\end{tabular}




\begin{tabular}{cccccccc}
\hline$T / \mathrm{K}$ & $10^{2} x$ & $\mathrm{SD}$ & $10^{2} x^{\text {cal,Apel }}$ & $T / \mathrm{K}$ & $10^{2} x$ & $\mathrm{SD}$ & $10^{2} x^{\text {cal,Apel }}$ \\
\hline 288.15 & 1.244 & $9.416 \mathrm{E}-06$ & 1.134 & 288.15 & 0.040 & $1.434 \mathrm{E}-05$ & 0.074 \\
293.15 & 1.734 & $1.838 \mathrm{E}-05$ & 1.607 & 293.15 & 0.057 & $2.205 \mathrm{E}-05$ & 0.106 \\
298.15 & 2.212 & $8.165 \mathrm{E}-07$ & 2.234 & 298.15 & 0.094 & $4.001 \mathrm{E}-05$ & 0.150 \\
303.15 & 2.972 & $2.002 \mathrm{E}-05$ & 3.051 & 303.15 & 0.114 & $2.748 \mathrm{E}-05$ & 0.210 \\
308.15 & 3.878 & $9.961 \mathrm{E}-05$ & 4.098 & 308.15 & 0.270 & $2.083 \mathrm{E}-05$ & 0.292 \\
313.15 & 5.204 & $5.062 \mathrm{E}-05$ & 5.417 & 313.15 & 0.367 & $8.190 \mathrm{E}-05$ & 0.402 \\
318.15 & 7.000 & $2.944 \mathrm{E}-06$ & 7.055 & 318.15 & 0.547 & $7.587 \mathrm{E}-05$ & 0.552 \\
323.15 & 9.567 & $6.220 \mathrm{E}-05$ & 9.058 & 323.15 & 0.846 & $9.226 \mathrm{E}-05$ & 0.751 \\
328.15 & 11.372 & $1.506 \mathrm{E}-04$ & 11.476 & 328.15 & 1.115 & $8.165 \mathrm{E}-05$ & 1.015 \\
333.15 & 14.272 & $3.838 \mathrm{E}-05$ & 14.353 & 333.15 & 1.269 & $8.288 \mathrm{E}-05$ & 1.364 \\
$\mathrm{n}-\mathrm{butanol}$ & & & & $\mathrm{n}-\mathrm{heptane}$ & & & \\
283.15 & 1.217 & $1.144 \mathrm{E}-05$ & 1.195 & 283.15 & 0.049 & $3.144 \mathrm{E}-05$ & 0.025 \\
288.15 & 1.491 & $2.944 \mathrm{E}-06$ & 1.547 & 288.15 & 0.049 & $2.944 \mathrm{E}-06$ & 0.044 \\
293.15 & 2.045 & $2.983 \mathrm{E}-05$ & 1.995 & 293.15 & 0.070 & $4.042 \mathrm{E}-05$ & 0.075 \\
298.15 & 2.464 & $4.082 \mathrm{E}-06$ & 2.564 & 298.15 & 0.099 & $1.960 \mathrm{E}-05$ & 0.121 \\
303.15 & 3.251 & $5.354 \mathrm{E}-06$ & 3.284 & 303.15 & 0.158 & $1.700 \mathrm{E}-05$ & 0.185 \\
308.15 & 4.065 & $1.245 \mathrm{E}-04$ & 4.191 & 308.15 & 0.269 & $6.492 \mathrm{E}-05$ & 0.272 \\
313.15 & 5.271 & $8.165 \mathrm{E}-07$ & 5.331 & 313.15 & 0.398 & $9.232 \mathrm{E}-05$ & 0.383 \\
318.15 & 6.800 & $5.838 \mathrm{E}-05$ & 6.760 & 318.15 & 0.525 & $8.822 \mathrm{E}-05$ & 0.518 \\
323.15 & 8.887 & $2.944 \mathrm{E}-06$ & 8.544 & 323.15 & 0.716 & $1.915 \mathrm{E}-04$ & 0.675 \\
328.15 & 10.777 & $0.000 \mathrm{E}+00$ & 10.766 & 328.15 & 0.819 & $1.004 \mathrm{E}-04$ & 0.850 \\
333.15 & 13.381 & $8.083 \mathrm{E}-05$ & 13.525 & 333.15 & 1.039 & $9.839 \mathrm{E}-05$ & 1.034 \\
\hline$x$
\end{tabular}

$x$ represents the experimentally measured average molar solubility; SD is the standard deviation of the average value according to the three parallel experiments; $x^{\text {cal }}$,Apel is the calculated solubility by the modified Apelblat equation. 
Table S2. Dissolving enthalpy $\left(\Delta_{\text {dis }} H\right)$ and dissolving entropy $\left(\Delta_{\text {dis }} S\right)$ in organic different solvent of 4-hexyloxybenzoic acid

\begin{tabular}{cccc}
\hline Solvent & $\Delta_{\text {dis }} \mathrm{H} / \mathrm{kJ} \cdot \mathrm{mol}^{-1}$ & $\Delta_{\text {dis }} \mathrm{S} / \mathrm{J} \cdot \mathrm{K}^{-1} \cdot \mathrm{mol}^{-1}$ & $\mathrm{R}^{2}$ \\
\hline ethyl acetate & 40.9860 & 107.5931 & 0.9957 \\
methanol & 44.4396 & 112.7309 & 0.99248 \\
ethanol & 38.4257 & 97.2387 & 0.98464 \\
2-propanol & 43.4373 & 114.3026 & 0.9984 \\
n-butanol & 38.2538 & 97.9153 & 0.99764 \\
n-hexane & 65.8025 & 163.3119 & 0.98442 \\
n-heptane & 53.8643 & 124.7055 & 0.98045 \\
acetonitrile & 40.3723 & 84.3543 & 0.9943 \\
\hline
\end{tabular}

Table S3. Dissolution Gibbs energy ( $\Delta$ dis G) and relative contribution of enthalpy $(\% \xi \mathrm{H})$ and entropy $(\% \xi \mathrm{S})$ of 4-hexyloxybenzoic acid

\begin{tabular}{cccccccc}
\hline$T / \mathrm{K}$ & $\Delta_{\text {dis }} G\left(\mathrm{~kJ} \cdot \mathrm{mol}^{-1}\right)$ & $\% \zeta H$ & $\% \zeta S$ & $T / \mathrm{K}$ & $\begin{array}{c}\Delta_{\text {dis } G} \\
\left(\mathrm{~kJ} \cdot \mathrm{mol}^{-1}\right)\end{array}$ & $\% \zeta H$ & $\% \zeta S$ \\
\hline methanol & & & & & \\
283.15 & 12.5198 & 58.1980 & 41.8020 & 283.15 & 10.5210 & 57.3624 & 42.6376 \\
288.15 & 11.9562 & 57.7715 & 42.2285 & 288.15 & 9.9830 & 56.9337 & 43.0663 \\
293.15 & 11.3925 & 57.3513 & 42.6487 & 293.15 & 9.4451 & 56.5114 & 43.4886 \\
298.15 & 10.8289 & 56.9371 & 43.0629 & 298.15 & 8.9071 & 56.0953 & 43.9047 \\
303.15 & 10.2652 & 56.5289 & 43.4711 & 303.15 & 8.3692 & 55.6853 & 44.3147 \\
308.15 & 9.7016 & 56.1265 & 43.8735 & 308.15 & 7.8312 & 55.2813 & 44.7187 \\
313.15 & 9.1379 & 55.7297 & 44.2703 & 313.15 & 7.2932 & 54.8831 & 45.1169 \\
318.15 & 8.5743 & 55.3386 & 44.6614 & 318.15 & 6.7553 & 54.4905 & 45.5095 \\
323.15 & 8.0106 & 54.9529 & 45.0471 & 323.15 & 6.2173 & 54.1036 & 45.8964 \\
328.15 & 7.4470 & 54.5725 & 45.4275 & 328.15 & 5.6793 & 53.7221 & 46.2779 \\
333.15 & 6.8833 & 54.1973 & 45.8027 & 333.15 & 5.1414 & 53.3459 & 46.6541 \\
ethanol & & & & acetonitrile & & & \\
283.15 & 10.8926 & 58.2571 & 41.7429 & 283.15 & 16.4874 & 62.8292 & 37.1708 \\
288.15 & 10.4064 & 57.8308 & 42.1692 & 288.15 & 16.0656 & 62.4195 & 37.5805 \\
293.15 & 9.9202 & 57.4107 & 42.5893 & 293.15 & 15.6438 & 62.0151 & 37.9849 \\
298.15 & 9.4340 & 56.9967 & 43.0033 & 298.15 & 15.2221 & 61.6159 & 38.3841 \\
303.15 & 8.9478 & 56.5886 & 43.4114 & 303.15 & 14.8003 & 61.2218 & 38.7782 \\
308.15 & 8.4616 & 56.1863 & 43.8137 & 308.15 & 14.3785 & 60.8327 & 39.1673 \\
313.15 & 7.9754 & 55.7897 & 44.2103 & 313.15 & 13.9568 & 60.4486 & 39.5514 \\
318.15 & 7.4892 & 55.3986 & 44.6014 & 318.15 & 13.5350 & 60.0692 & 39.9308 \\
323.15 & 7.0030 & 55.0130 & 44.9870 & 323.15 & 13.1132 & 59.6946 & 40.3054 \\
\hline & & & & & & &
\end{tabular}




\begin{tabular}{|c|c|c|c|c|c|c|c|}
\hline$T / \mathrm{K}$ & $\Delta_{\mathrm{dis}} G\left(\mathrm{~kJ} \cdot \mathrm{mol}^{-1}\right)$ & $\% \zeta H$ & $\% \zeta S$ & $T / \mathrm{K}$ & $\begin{array}{c}\Delta_{\mathrm{dis}} G \\
\left(\mathrm{~kJ} \cdot \mathrm{mol}^{-1}\right)\end{array}$ & $\% \zeta H$ & $\% \zeta S$ \\
\hline 328.15 & 6.5168 & 54.6327 & 45.3673 & 328.15 & 12.6914 & 59.3246 & 40.6754 \\
\hline 333.15 & 6.0306 & 54.2577 & 45.7423 & 333.15 & 12.2697 & 58.9592 & 41.0408 \\
\hline 2-propanol & & & & n-hexane & & & \\
\hline 283.15 & 11.0725 & 57.3036 & 42.6964 & 283.15 & 19.5607 & 58.7290 & 41.2710 \\
\hline 288.15 & 10.5010 & 56.8748 & 43.1252 & 288.15 & 18.7442 & 58.3041 & 41.6959 \\
\hline 293.15 & 9.9295 & 56.4523 & 43.5477 & 293.15 & 17.9276 & 57.8853 & 42.1147 \\
\hline 298.15 & 9.3580 & 56.0361 & 43.9639 & 298.15 & 17.1111 & 57.4725 & 42.5275 \\
\hline 303.15 & 8.7865 & 55.6260 & 44.3740 & 303.15 & 16.2945 & 57.0655 & 42.9345 \\
\hline 308.15 & 8.2150 & 55.2218 & 44.7782 & 308.15 & 15.4779 & 56.6642 & 43.3358 \\
\hline 313.15 & 7.6434 & 54.8235 & 45.1765 & 313.15 & 14.6614 & 56.2686 & 43.7314 \\
\hline 318.15 & 7.0719 & 54.4309 & 45.5691 & 318.15 & 13.8448 & 55.8784 & 44.1216 \\
\hline 323.15 & 6.5004 & 54.0438 & 45.9562 & 323.15 & 13.0283 & 55.4936 & 44.5064 \\
\hline 328.15 & 5.9289 & 53.6623 & 46.3377 & 328.15 & 12.2117 & 55.1141 & 44.8859 \\
\hline 333.15 & 5.3574 & 53.2860 & 46.7140 & 333.15 & 11.3951 & 54.7397 & 45.2603 \\
\hline n-butanol & & \multicolumn{6}{|c|}{ n-heptane } \\
\hline 283.15 & 10.5291 & 57.9792 & 42.0208 & 283.15 & 18.5539 & 60.4031 & 39.5969 \\
\hline 288.15 & 10.0395 & 57.5521 & 42.4479 & 288.15 & 17.9304 & 59.9837 & 40.0163 \\
\hline 293.15 & 9.5499 & 57.1313 & 42.8687 & 293.15 & 17.3069 & 59.5701 & 40.4299 \\
\hline 298.15 & 9.0604 & 56.7166 & 43.2834 & 298.15 & 16.6834 & 59.1621 & 40.8379 \\
\hline 303.15 & 8.5708 & 56.3079 & 43.6921 & 303.15 & 16.0598 & 58.7597 & 41.2403 \\
\hline 308.15 & 8.0812 & 55.9050 & 44.0950 & 308.15 & 15.4363 & 58.3627 & 41.6373 \\
\hline 313.15 & 7.5916 & 55.5079 & 44.4921 & 313.15 & 14.8128 & 57.9711 & 42.0289 \\
\hline 318.15 & 7.1020 & 55.1163 & 44.8837 & 318.15 & 14.1892 & 57.5846 & 42.4154 \\
\hline 323.15 & 6.6125 & 54.7303 & 45.2697 & 323.15 & 13.5657 & 57.2033 & 42.7967 \\
\hline 328.15 & 6.1229 & 54.3496 & 45.6504 & 328.15 & 12.9422 & 56.8270 & 43.1730 \\
\hline 333.15 & 5.6333 & 53.9742 & 46.0258 & 333.15 & 12.3187 & 56.4556 & 43.5444 \\
\hline
\end{tabular}

Table S4. The polymorph outcome of crash cooling crystallization

\begin{tabular}{|c|c|c|c|c|}
\hline Solvent & Temperature & Supersaturation & Induction time & Form \\
\hline \multirow{10}{*}{ ethanol } & \multirow{10}{*}{$15^{\circ} \mathrm{C}$} & \multirow{5}{*}{1.3} & $2 \min 48 \mathrm{~s}$ & $\mathrm{I}$ \\
\hline & & & $4 \min$ & $\mathrm{I}$ \\
\hline & & & $3 \min 12 \mathrm{~s}$ & $\mathrm{I}$ \\
\hline & & & $4 \min 58 \mathrm{~s}$ & I \\
\hline & & & $4 \min 46 s$ & I \\
\hline & & \multirow{5}{*}{1.50} & $1 \mathrm{~min} 30 \mathrm{~s}$ & $\mathrm{I}$ \\
\hline & & & $1 \min 14 \mathrm{~s}$ & II \\
\hline & & & $2 \min$ & $\mathrm{I}$ \\
\hline & & & $1 \mathrm{~min} 28 \mathrm{~s}$ & II \\
\hline & & & $1 \min 17 \mathrm{~s}$ & II \\
\hline
\end{tabular}




\begin{tabular}{|c|c|c|c|c|}
\hline & & & $2 \mathrm{~min}$ & I \\
\hline & & \multirow{5}{*}{1.7} & $37 \mathrm{~s}$ & $\mathrm{I}$ \\
\hline & & & $30 \mathrm{~s}$ & I \\
\hline & & & $28 \mathrm{~s}$ & $\mathrm{I}$ \\
\hline & & & $39 \mathrm{~s}$ & I \\
\hline & & & $36 \mathrm{~s}$ & I \\
\hline & & \multirow{5}{*}{2} & $50 \mathrm{~s}$ & $\mathrm{I}$ \\
\hline & & & $54 \mathrm{~s}$ & I \\
\hline & & & $60 \mathrm{~s}$ & I \\
\hline & & & $60 \mathrm{~s}$ & $\mathrm{I}$ \\
\hline & & & $60 \mathrm{~s}$ & $\mathrm{I}$ \\
\hline & & \multirow{5}{*}{2.50} & instantly & $\mathrm{I}$ \\
\hline & & & $3 \mathrm{~s}$ & I \\
\hline & & & $3 \mathrm{~s}$ & I \\
\hline & & & $3 \mathrm{~s}$ & I \\
\hline & & & $3 \mathrm{~s}$ & I \\
\hline & \multirow{25}{*}{$30^{\circ} \mathrm{C}$} & \multirow{5}{*}{1.3} & $3 \min 40 \mathrm{~s}$ & I \\
\hline & & & $3 \min 14 s$ & $\mathrm{I}$ \\
\hline & & & $3 \min$ & I \\
\hline & & & $2 \mathrm{~min}$ & I \\
\hline & & & $5 \mathrm{~min}$ & $\mathrm{I}$ \\
\hline & & \multirow{5}{*}{1.5} & $24 \mathrm{~s}$ & $\mathrm{I}$ \\
\hline & & & $1 \mathrm{~min} 40 \mathrm{~s}$ & I \\
\hline & & & $2 \min 30 \mathrm{~s}$ & I \\
\hline & & & $30 \mathrm{~s}$ & I \\
\hline & & & $20 \mathrm{~s}$ & I \\
\hline & & \multirow{5}{*}{1.7} & $10 \mathrm{~s}$ & I \\
\hline & & & $9 \mathrm{~s}$ & I \\
\hline & & & $10 \mathrm{~s}$ & I \\
\hline & & & $6 \mathrm{~s}$ & I \\
\hline & & & $13 \mathrm{~s}$ & I \\
\hline & & \multirow{3}{*}{2} & $5 \mathrm{~s}$ & I \\
\hline & & & $4 \mathrm{~s}$ & I \\
\hline & & & instantly & I \\
\hline & & \multirow{7}{*}{2.2} & $4 \mathrm{~s}$ & I \\
\hline & & & $3 \mathrm{~s}$ & I \\
\hline & & & instantly & I \\
\hline & & & instantly & I \\
\hline & & & instantly & I \\
\hline & & & instantly & I \\
\hline & & & instantly & I \\
\hline \multirow{2}{*}{ 2-propanol } & \multirow{2}{*}{$15^{\circ} \mathrm{C}$} & \multirow{2}{*}{1.2} & $16 \min$ & I \\
\hline & & & $13 \mathrm{~min}$ & I \\
\hline
\end{tabular}




\begin{tabular}{|c|c|c|c|c|}
\hline & & & $14 \mathrm{~min}$ & $\mathrm{I}$ \\
\hline & & & $15 \mathrm{~min}$ & $\mathrm{I}$ \\
\hline & & & $15 \mathrm{~min}$ & $\mathrm{I}$ \\
\hline & & & $1 \mathrm{~min} 52 \mathrm{~s}$ & I \\
\hline & & & $1 \min 56 \mathrm{~s}$ & I \\
\hline & & 1.43 & $49 \mathrm{~s}$ & $\mathrm{I}$ \\
\hline & & & $50 \mathrm{~s}$ & $\mathrm{I}$ \\
\hline & & & $45 \mathrm{~s}$ & $\mathrm{I}$ \\
\hline & & & instantly & I \\
\hline & & 1.87 & instantly & $\mathrm{I}$ \\
\hline & & & instantly & $\mathrm{I}$ \\
\hline & & & instantly & $\mathrm{I}$ \\
\hline & & & instantly & I \\
\hline & & & instantly & $\mathrm{I}$ \\
\hline & & & instantly & I、 II \\
\hline & & 2.28 & instantly & I、II \\
\hline & & & instantly & I、 II \\
\hline & & & instantly & I、II \\
\hline & & & $11 \mathrm{~min}$ & $\mathrm{I}$ \\
\hline & & 110 & $25 \mathrm{~min}$ & $\mathrm{I}$ \\
\hline & & 1.10 & $45 \mathrm{~min}$ & I \\
\hline & & & $30 \mathrm{~min}$ & $\mathrm{I}$ \\
\hline & & & $72 s$ & I \\
\hline & & & $48 \mathrm{~s}$ & $\mathrm{I}$ \\
\hline & & 1.31 & $75 \mathrm{~s}$ & II \\
\hline & & & $76 \mathrm{~s}$ & I \\
\hline & & & $49 \mathrm{~s}$ & $\mathrm{I}$ \\
\hline & $30^{\circ} \mathrm{C}$ & & instantly & $\mathrm{I}$ \\
\hline & & & instantly & $\mathrm{I}$ \\
\hline & & 2.00 & instantly & $\mathrm{I}$ \\
\hline & & & instantly & $\mathrm{I}$ \\
\hline & & & instantly & I \\
\hline & & & instantly & II \\
\hline & & & instantly & II \\
\hline & & 2.21 & instantly & $\mathrm{I}$ \\
\hline & & & instantly & II \\
\hline & & & instantly & II \\
\hline & & & $20 \mathrm{~min}$ & I \\
\hline & & 130 & $5 \min 10 \mathrm{~s}$ & $\mathrm{I}$ \\
\hline ethyl ocetate & $15^{\circ} \mathrm{C}$ & 1.50 & $3 \min 20 \mathrm{~s}$ & $\mathrm{I}$ \\
\hline enyi acetale & 10 & & $3 \min 27 \mathrm{~s}$ & $\mathrm{I}$ \\
\hline & & 150 & $50 \mathrm{~s}$ & $\mathrm{I}$ \\
\hline & & 1.50 & $50 \mathrm{~s}$ & I \\
\hline
\end{tabular}




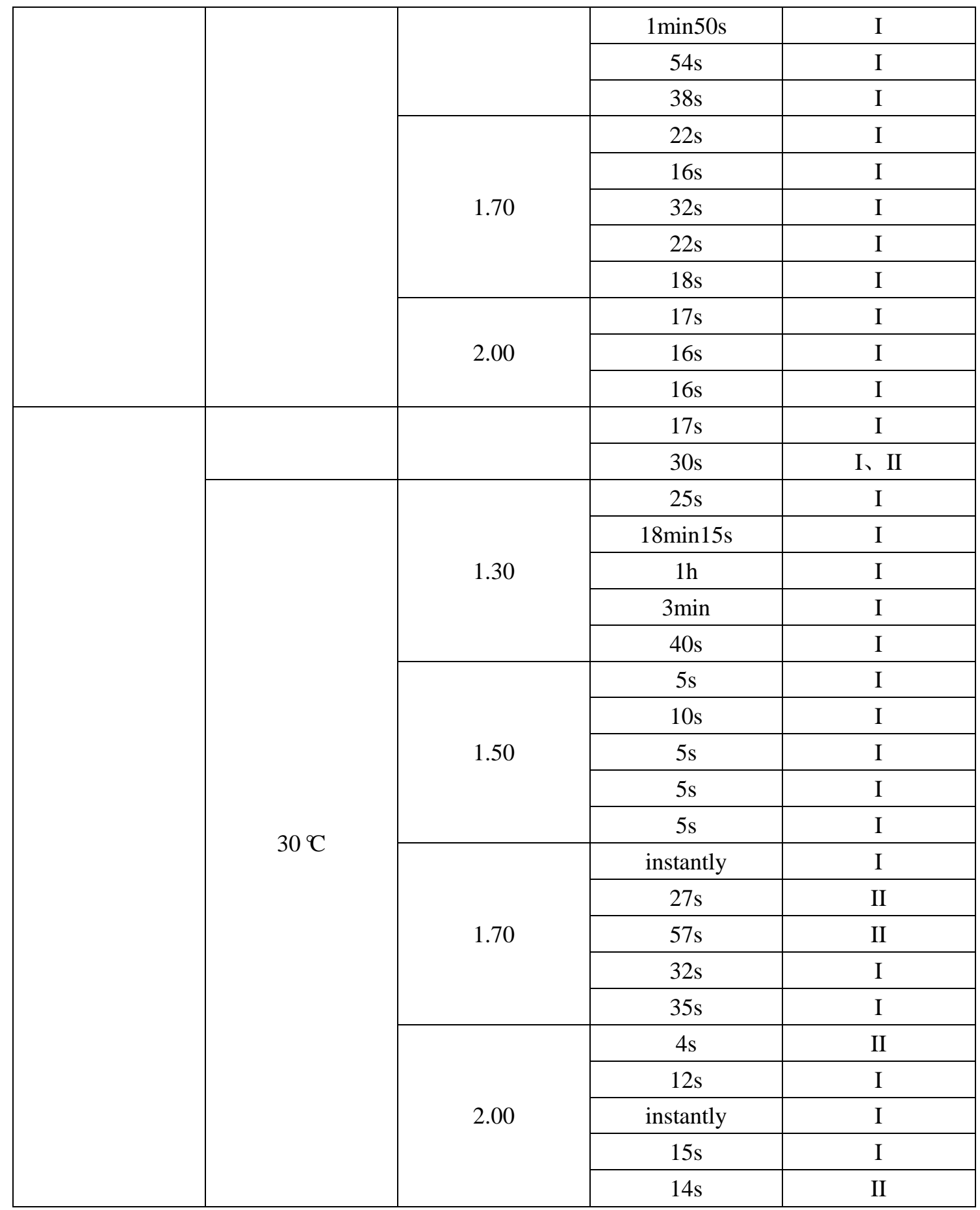


Table S5. The polymorph outcome of evaporative crystallization

\begin{tabular}{|c|c|c|c|c|c|}
\hline solvent & $\begin{array}{c}T \\
\left({ }^{\circ} \mathrm{C}\right)\end{array}$ & $\begin{array}{l}\text { Vacuum } \\
\text { degree } \\
\text { (MPa) }\end{array}$ & $\begin{array}{l}\text { Induction } \\
\text { time } \\
\text { (s) }\end{array}$ & $\begin{array}{c}\text { Sampling Time } \\
\text { (s) }\end{array}$ & $\begin{array}{c}\text { Polymorph } \\
\text { Outcome }\end{array}$ \\
\hline \multirow{4}{*}{ methanol } & 20 & 0.1 & 90 & 304 & I and II \\
\hline & 30 & 0.1 & 46 & 102 & II \\
\hline & \multirow{2}{*}{50} & 0.068 & 0 & & II \\
\hline & & 0.076 & 0 & & II \\
\hline \multirow{4}{*}{ ethanol } & 20 & 0.1 & 95 & 2640 & II \\
\hline & 30 & 0.1 & 86 & 311 & II \\
\hline & \multirow{2}{*}{60} & 0.075 & 0 & & II \\
\hline & & 0.070 & 0 & & II \\
\hline \multirow{4}{*}{ 2-propanol } & 20 & 0.1 & $>8 \mathrm{~h}$ & & \\
\hline & 30 & 0.1 & 88 & 480 & II \\
\hline & \multirow{2}{*}{60} & 0.080 & 0 & & II \\
\hline & & 0.085 & 0 & & II \\
\hline \multirow{4}{*}{$\begin{array}{c}\text { ethyl } \\
\text { acetate }\end{array}$} & 20 & 0.1 & 81 & 261 & II \\
\hline & 30 & 0.1 & 56 & 122 & II \\
\hline & \multirow{2}{*}{60} & 0.069 & 0 & & II \\
\hline & & 0.075 & 0 & & II \\
\hline
\end{tabular}


Table S6. The polymorph outcomes of anti-solvent crystallization

\begin{tabular}{|c|c|c|c|c|}
\hline solvent & $\begin{array}{c}T \\
\left({ }^{\circ} \mathrm{C}\right)\end{array}$ & $\begin{array}{c}\text { Molar ratio } \\
\text { (solvent to anti-solvent) }\end{array}$ & $\begin{array}{l}\text { Induction time } \\
\text { (s) }\end{array}$ & $\begin{array}{l}\text { Polymorph } \\
\text { Outcome }\end{array}$ \\
\hline \multirow{4}{*}{ ethanol } & \multirow{2}{*}{15} & $1: 2.68$ & 0 & II \\
\hline & & $1: 4.48$ & 0 & II \\
\hline & \multirow{2}{*}{30} & $1: 2.81$ & 0 & II \\
\hline & & $1: 4.69$ & 0 & II \\
\hline \multirow{4}{*}{ 2-propanol } & \multirow{2}{*}{15} & $1: 3.50$ & 0 & II \\
\hline & & $1: 5.84$ & 0 & II \\
\hline & \multirow{2}{*}{30} & $1: 3.67$ & 0 & II \\
\hline & & $1: 6.12$ & 0 & II \\
\hline
\end{tabular}

